SOME INTERESTING SEQUELE OF A CASE OF SCARLET FEVER.

Read before the Philadelphia County Medical Society, on Fanuary 25, 1888 .

BY A. J. DOWNES, M.D., OF PHILADELYHIA, PA. the boy, although not well for a few years back, was comparatively so. For two weeks previous to his death he was confined to his bed, and undoubtedly during this time he was suffering from a glomerulonephritis which, adding itself to an already damaged kidney, precipitated an attack of uræmia.

Additional proof that the boy's kidneys were not

On November I5, I887, at 3 P.M., I was asked to normal prior to his last sickness is not wanting. His see B. M., a boy of 7 years, then under the care of bladder, post-mortem, contained about two ounces a homcopath, and said to be dying of heart disease. I called immediately. The boy was comatose, had been so for twelve hours. The face had a characteristic pallor, the eye-lids were slightly puffy, the pupils dilated, the breathing stertorous.

I listened to the heart and heard a loud systolic apex murmur, a diastolic one at the right base, and mingling with both a peculiar whistling sound, the cause of which at the time I did not understand. Examining further, I found that the eyelids had become puffy two days previous, that the feet and ankles were now swollen, and the scrotum slightly so. No urine had passed in twenty-four hours. Excepting a few scybala, forty-eight hours before, the bowels had not moved in seventy-two hours.

The child had scarlet fever five years before. I told the father that the boy did have heart disease, but that he was dying of uramia. Shortly after 5 P.M. the boy was dead. The following morning Dr. Martin Rively and myself made a postmortem examination. The specimens I show are the heart, kidneys and a piece of the liver.

So advanced cardiac lesions in a child of this age are far from common. The ventricles are both hypertrophied and the cavities dilated, the right considerably. The curtains of the mitral valve are thickened and fibrous, the posterior leaflet is exceedingly contracted and shows above it a recent inflammatory area. The aortic curtains are a beautiful picture of an insufficient valve. The whistling sound I heard during life must have come from the tricuspid valve. The hypertrophy of the muscular elements of the ventricle, with the marked distention to which the cavity was subjected, surely allowed sufficient leakage to cause this sound.

On account of its so late recognition and its undoubted influence in enhancing the cardiac condition the state of the kidneys is exceedingly interesting. Even microscopically the kidneys show changes which must have been going on for some time. The pale cortex and the eversion of the cut edges indicate fat. Under the microscope we find evidence of long existing changes, sufficient to warrant the assumption that in the long-ago attack of scarlet fever the kidney changes began. The tubules are swollen, distorted, and full of casts. The epithelial cells lining them are somewhat granular and smaller than normal with their nuclei becoming indistinct. Long existing changes therefore. We find also recent conditions.

The glomerules are swollen and congested and in their vicinity an infiltration of small indifferent cells. A very characteristic glomerulo-nephritis of recent date. The cause in this case of anuria and consequent uræmia. And the history points to this: for -twenty-fur hours' urine-of this I collected one ounce. It contained a large amount of albumen but no sugar, round and columnar epithelial cells showing fatty degeneration, a few epithelial casts containing oil globules and some free oil. A few weeks nephritis could not cause this urine.

The liver changes are secondary to the heart condition. Under this microscope it shows both fatty infiltration and degeneration.

In concluding, I would recall the points that interested me in the case. First. The extensive cardiac lesions caused by scarlet fever, occurring in a child under 2 years. Second. The late recognition of the heart, the non-recognition of the kidney condition. Third. The occurrence of acute inflammation-the glomerulo-nephritis almost typical of scarlet fever-in an already damaged kidney. Finally. May I not call attention to this case as showing to what extent the sequela of scarlet fever may advance when not recognized and treated. And the history of the case proves such to be the fact. Eight homœopaths in succession had charge of the boy for four years prior to his death. Over a year ago one had diagnosticated diabetes. The one in attendance before my visits had recognized valvular affection. But the kidney lesions had escaped them all. Apparently this proves that the case ran an almost natural course.

\section{ABSCESS OF THE CEREBELLUM CAUSED BY DISEASE OF THE EAR.}

BY THOMAS C. SMITH, M.D., OF WASHINGTON, D. C.

Disease of the brain caused by the neglect of middle-ear affections, is by no means uncommon, and the case to be reported is another instance of the fallacy of supposing that a chronic otorrhœa is best treated by being let alone.

On August 29,1887 , I was asked to see $M$. W., colored, at. I 5 years. When an infant she had an attack of measles, following which came a discharge from the ear and almost complete loss of hearing. She was always sick. Headache was a frequent affair, and at the time of my visit she was experiencing an unusually severe attack. There was some fever; constipation; loss of appetite; rather slow pulse. Bromide of potassium was given for the headache, and the bowels were moved by suitable means. At my next visit I learned that the girl had had convulsions after my visit, and had passed a sleepless night. I now examined the ears and found the left meatus filled with pus. Her mother informed me that she had suffered from the ear disease, with dis- 
charge, ever since the attack of measles, as already mentioned. At times the discharge of pus would cease for a while but her symptoms did not seem to be modified thereby. She did not think anything could be done for the ear disease, consequently, nothing had been done. The mother was given to understand that the headache and convulsions were due to the ear disease and an unfavorable prognosis was given. The ear was syringed and an astringent used to try to influence the diseased middle-ear tissues. Chloral at night secured sleep, and after a week my visits were discontinued. On September 14, the girl came to my office with her mother, and the earwas examined. The purulent discharge was not quite so free, but the meatus was so much obstructed by granulation-tissue that it was impossible to get a view of the deep portion of that tube. The general condition of the girl was so bad that I did not think any treatment would avail much. Still, she was ordered tonics and good nourishment, and the ear was to be cleansed with astringent and antiseptic remedies. I did not see her again until the 12 th of the present month. She was in bed suffering from headache. On the 14th she was easier, but she was disposed to be drowsy. On the $5^{\text {th }}$ coma existed and she died about the middle of the day. The diagnosis of intracranial abscess had been made. No paralytic symptoms existed at any time.

The case is interesting from a surgical point of view. Brain surgery is making such great advances, that the time may come when all intracranial abscesses and tumors will be subjected to the same treatment as when they occur in other locations. In view of the fact that the existence of abscess was so clearly established in this case, the question arises, Why was not the skull trephined and the abscess sought out and opened? In the light of the symptoms presented, I answer the question by asking another. Where would the surgeon have applied his trephine? And with the post-mortem evidence before us, I ask whether an operation would have been beneficial even supposing the abscess to have been correctly located before the patient was in extremis?

The autopsy was made by Dr. Lamb, and I append his notes:

"Necroscopy.-Head alone examined. Left meatus auditorious externus occupied by thick, purulent matter of dark, rather bloody hue. Dura mater normal, except on posterior surface of petrous portion of left temporal bone, where the adhesion was a little greater than normal around an opening in the bone; this opening was one-quarter of an inch in diameter and communicated with the tympanic cavity; this cavity, again, was enlarged by progressive excavation of the bone, into a chamber an inch in length, and of corresponding breadth and height. Cerebrum normal. Left hemisphere of cerebellum, anterior half converted into an abscess."

Kaschan Hospital. - A few years ago three Russian female physicians founded a hospital at Kaschan, Persia, for Mussulman women. The experiment has met with great success, and in the last twelve months 15,000 patients have been treated.

\section{MEDICAL PROGRESS.}

The Telephonic Bullet-Probe. - At the meeting of the Surgical Section of the New York Academy of Medicine on January 9, DR. J. H. GIRDNER read a paper on this subject. In the past year he has perfected the instrument that he described a year ago, and now operates it by a current of electricity extracted from the body of the patient himself. This instrument, as now arranged, leaves both hands of the operator free, and shuts out all sound except that heard when the bullet is touched. Dr. Girdner reports the following case :

A musket-ball had lain between the tibia and fibula for twenty-two years. A long, narrow, tortuous sinus had been discharging for a year. When an ordinary probe was passed, hard substances could be felt in many places; but you could not tell if bone or bullet was being probed. The porcelain-probe could not have been marked by the lead owing to thick crusts of salts of lead with which the ball was covered, even if it could have been brought into contact with the bullet, which it could not, owing to narrow places in the sinus. Bone and other tissue were felt as the probe passed to different parts of the wound, but no response was heard in the telephone until the leaden bullet was touched, then an electric current passed through the telephone; and as often as this current was made and broken, by touching and removing the probe from the lead, so often was there a vibration of the diaphragm, and consequently a clicking and scraping sound heard in the telephone; in other words, the patient's body was converted into an electric battery; the body corresponded to the cups, its fluids and heat to the battery fluid, the steel bulb immersed in the mouth to the zinc, let us say, and the lead when it was touched, to the carbon, and thus our battery was completed, a current obtained, and the metal diaphragn made to vibrate.

The advantages of this instrument over all others at once appear, when it is remembered, that in its use the accurate sense of hearing is substituted for that of the sensation communicated to the hand, which is always unreliable, for no one can tell if a hard substance felt in a wound be bone, metal, or some other hard tissue. The porcelain-tipped probe was made with the hope of overcoming this difficulty; but after ample experience with the Nélaton probe, both in my own hands and in those of others in my presence, I am certain that, unless the bullet is perfectly clean from grease, lead salts, etc., and very favorably situated, it is not possible to obtain lead markings on the porcelain tip which can be relied upon to direct our operative procedure. Let any one hold a bullet in the hand, and probe it with a Nélaton probe until the markings of the lead on the porcelain point are perfectiy distinct, and he will find that it requires an amount of force and pressure in rubbing the lead which he will rarely be able to make, even in the most favorable cases of gunshot-wound. None of the above conditions, which make the Nélaton probe useless, in any way interfere with the perfect working of this new probe, for the slightest touch of the bullet 